\title{
Patrick Fournier et Geneviève Massard-Guilbaud (dir.), Aménagement et environnement. Perspectives historiques
}

Rennes, Presses universitaires de Rennes, 2016

\section{Charles-François Mathis}

\section{OpenEdition}

\section{Journals}

Édition électronique

URL : http://journals.openedition.org/artefact/917

DOI : $10.4000 /$ artefact. 917

ISSN : 2606-9245

Éditeur :

Association Artefact. Techniques histoire et sciences humaines, Presses universitaires du Midi

Édition imprimée

Pagination : 219-221

ISBN : 978-2-7535-7305-5

ISSN : 2273-0753

Référence électronique

Charles-François Mathis, « Patrick Fournier et Geneviève Massard-Guilbaud (dir.), Aménagement et environnement. Perspectives historiques », Artefact [En ligne], 6 | 2017, mis en ligne le 31 mai 2018, consulté le 24 septembre 2020. URL : http://journals.openedition.org/artefact/917 ; DOI : https:// doi.org/10.4000/artefact.917

Ce document a été généré automatiquement le 24 septembre 2020.

\section{(i) $९$

Artefact, Techniques, histoire et sciences humaines est mise à disposition selon les termes de la Licence Creative Commons Attribution - Pas d'Utilisation Commerciale - Pas de Modification 4.0 International. 


\title{
Patrick Fournier et Geneviève Massard-Guilbaud (dir.), Aménagement et environnement. Perspectives historiques
}

Rennes, Presses universitaires de Rennes, 2016

\author{
Charles-François Mathis
}

\section{RÉFÉRENCE}

Patrick Fournier et Geneviève Massard-Guilbaud (dir.), Aménagement et environnement.

Perspectives historiques, Rennes, Presses universitaires de Rennes, 2016, 300 p.

1 Les controverses et les événements tragiques entourant la construction de l'aéroport de Notre-Dame-des-Landes sont un exemple contemporain criant des problèmes soulevés par des perspectives d'aménagements. S'y trouvent en jeu, en effet, les conséquences environnementales $\mathrm{du}$ projet, mais aussi son coût économique, la légitimité démocratique des acteurs, les choix de société sous-jacents, etc.

Ce sont justement ces questionnements qui se trouvent au cœur de l'ouvrage collectif dirigé par Patrick Fournier et Geneviève Massard-Guilbaud. Issu d'un colloque organisé par le Réseau universitaire de chercheurs en histoire environnementale (RUCHE), il témoigne de la vitalité de ce champ de recherches et de ses apports. Comme l'indique P. Fournier dans sa remarquable introduction, elle permet en effet de sortir d'une histoire de l'aménagement par le haut, processus surplombant mené par l'État, à l'instigation d'élites éclairées, dans une vision techniciste de l'espace. Au contraire, l'aménagement est perçu alors comme un processus d'interactions, de conflits et de négociations, au cœur d'écosystèmes, mettant en jeu dès lors des acteurs multiples, $\mathrm{y}$ compris "non-humains", et sur un temps plus long. Les dix-sept interventions d'historiens, archéologues et géographes de cet ouvrage, centrées sur l'Europe mais 
avec des aperçus sur des territoires coloniaux ou sur les États-Unis, permettent d'éclairer cette problématique. Elles sont organisées en trois ensembles: "Construire les territoires, transformer l'environnement »; «Endiguer, canaliser : le cours d'eau, vecteur des aménagements territoriaux»; les impacts socio-environnementaux de l'aménagement urbain et portuaire. Mais, pour mieux dégager les enjeux et enseignements qui s'y dévoilent, nous ne tiendrons pas compte ici de cette tripartition.

3 Le premier élément frappant est tout simplement l'ampleur des conséquences environnementales des aménagements : c'est particulièrement perceptible lorsque des cours d'eau sont concernés. Dominique Baud, Jonathan Bussard et Emmanuel Reynard mettent ainsi très clairement en évidence le «fort impact environnemental » de la correction du Rhône valaisan au XIX ${ }^{e}$ siècle, avec des effets sur la couverture du sol et des modifications paysagères. Jacky Girel montre la même chose en ce qui concerne l'aménagement de l'Isère, qui s'enfonce dans ses alluvions à la suite de la construction de barrages, de la correction des torrents, etc. De la même manière, le curage de la Reyssouze, étudié par Alexandre Brun, Hervé Caltran, Stéphane Coursière et Stéphane Frioux, entraîne un appauvrissement biologique de la rivière.

4 Ces dommages environnementaux, en général non anticipés bien sûr, sont souvent accompagnés de conséquences sociales fortes pour les populations : la canalisation de la Vilaine, présentée par Katherine Dana, qui se met en place très tôt, au Xvi siècle, a d'abord des objectifs économiques qui prennent peu ou pas en compte les intérêts des riverains. De même, comme le montrent Marie Fournier et Nicolas Holleville, la mise en place d'un canal de décharge de la rivière Ill, à Mulhouse, vise d'abord à lutter contre les inondations, mais répond à d'autres espérances également: assainissement des marécages pour récupérer et valoriser le foncier, assurer du travail à des populations pauvres en nombre grandissant; l'organisation de la ville elle-même s'en trouve évidemment profondément modifiée, ce canal contribuant également à une forme de ségrégation sociale.

5 Le recours à la puissance publique peut sembler alors une évidence, même s'il n'a pas toujours les retombées heureuses que l'on en attend. Dans le cas des usines de raffinage du sucre installées à Orléans et La Rochelle, présentées par Gaëlle Caillet, les autorités, particulièrement au $\mathrm{xIx}^{\mathrm{e}}$ siècle, ont tendance à privilégier le développement économique au détriment des citadins incommodés par les nuisances considérables de ces installations. Ces usages contradictoires se retrouvent même dans le cas d'un espace plus naturel, comme la forêt de la Sainte-Baume, dont Martine Chalvet montre bien qu'elle est un lieu d'interactions entre des aménagements anthropiques et un écosystème forestier dont les échelles spatiales et temporelles sont différentes et où la multifonctionnalité de la forêt amène à des choix qui sont, fondamentalement, des choix de société. L'existence et l'intervention d'une autorité régulatrice sont pourtant souvent requises : Raphaël Morera souligne, dans le cas de la gestion de l'eau dans le Calaisis au tournant des $\mathrm{XVII}^{\mathrm{e}}$ et $\mathrm{XVII} \mathrm{e}^{\mathrm{e}}$ siècles, que, face à la concurrence que se livrent autorités militaires et civiles, les populations locales s'en remettent à l'autorité royale pour qu'elle arbitre. Par ailleurs, ici, comme pour nombre d'aménagements, particulièrement concernant les aspects hydrauliques, une constance est nécessaire dans l'entretien des installations : il importe donc qu'une autorité de gestion soit mise en place, ce qui est rarement le cas. À Padoue, entre les $\mathrm{XII}^{\mathrm{e}}$ et $\mathrm{XV}^{\mathrm{e}}$ siècles, c'est, entre autres, l'absence d'un tel instrument de gestion qui explique l'échec de la lutte contre les inondations, comme le montre Rémy Simonetti. 
6 Les échecs sont donc nombreux, mais il y a là bien entendu un biais d'historiens qui s'en préoccupent plus que des succès. Néanmoins, certaines contributions mettent en évidence le rôle heuristique de certaines déconfitures. La plus emblématique de toutes est sans doute celle de l'arsenal de Rochefort, qui devait être une vitrine du pouvoir de Louis XIV. Les difficultés suscitées par l'environnement particulier du site et dont on ne prend conscience qu'au fur et à mesure de l'aménagement, entraînent à terme sa relégation, au profit de Brest. Mais, comme le suggère Sébastien Martin, on peut parler ici d'un «brouillon grandeur nature " qui permet par la suite d'éviter de tels empêchements dans les ports militaires du sud de la France. De la même manière, Stéphane Durand montre que les conséquences environnementales fortes des aménagements portuaires en Languedoc aux XVII ${ }^{e}$ et XVIII ${ }^{e}$ siècles finissent par susciter des interrogations sur les mécanismes naturels pour tâcher de les comprendre et de s'en accommoder. En Guyane, dans la première moitié du XIX siècle, et contrairement à ce que l'on croyait, les administrateurs étudiés par Jean-Yves Puyo ont recours à des expertises solides et nombreuses avant d'intervenir dans l'aménagement de la colonie sans échapper à de cuisantes déconvenues.

7 Ces interrogations montrent l'intérêt de s'insérer dans un temps long où l'apprentissage peut se faire et se défaire. C'est ce que proposent Romana Harfouche et ses confrères dans un chapitre sur l'aménagement des montagnes du Liban depuis l'Antiquité, qui font l'objet d'un aménagement ancien, sans plan préconçu, dans une adaptation constante et réciproque des sociétés et de leur environnement, jusqu'à ce que la rapidité des bouleversements de la période contemporaine la rende plus difficile. C'est en tout cas l'absence de linéarité qui frappe ici comme ailleurs : l'aménagement n'est pas un processus continu, téléologique, il est fait d'arrêts, de relances, d'accélérations, de retours en arrière parfois. L'un des enseignements du bel article d'Elsa Devienne est que la configuration actuelle des plages de Los Angeles ne résulte pas d'un projet préconçu, mais est d'abord la conséquence des modifications environnementales provoquées par la création du port de plaisance de Santa Monica. Cette élaboration au fil du temps n'est d'ailleurs pas synonyme d'échec, puisqu'on a là un des rares cas où un aménagement pourtant lourd semble positif tant socialement que du point de vue environnemental.

8 Un dernier fil rouge qui nous semble courir à travers cet ouvrage passionnant est celui du poids des représentations dans la mise en œuvre de certains aménagements et de leur évaluation. Anne-Marie Granet-Abisset met ainsi bien en évidence comment se construit un "terrain de jeu alpin» qui fait entrer en contradiction de multiples exigences touristiques, vivrières, économiques, sociales, environnementales, etc. En particulier, l'aménagement des espaces montagnards au bénéfice des touristes est une conséquence d'une montagne imaginée, celle des citadins, qui la façonnent ainsi au risque d'une muséification. Le fleuve Los Angeles lui-même, étudié par Hélène Schmutz, voit ses visages varier au fil du temps selon les évolutions sociales et culturelles et les besoins qu'on en a. Enfin, à Bruxelles, Odile de Bruyn met bien en évidence que le développement de parcs et squares par le roi Léopold II sur le modèle parisien se heurte à de fortes résistances faute d'avoir pris en compte une identité nationale en construction qui voulait préserver un certain héritage paysager et naturel. Mais le temps passant, ces modifications sont plus acceptées : la nature change, les Bruxellois aussi. 
9 C'est qu'il y a bien, comme l'affirme avec justesse Geneviève Massard-Guilbaud dans sa postface, une ambiguité des notions de succès ou d'échec: le temps peut les inverser, les points de vue aussi peuvent varier sur un même aménagement. Surtout, et l'on ne peut que s'accorder avec cette historienne, ce n'est pas à l'histoire de juger : à elle le soin de poser des questions pertinentes, de fournir les connaissances permettant de réfléchir et d'embrasser toute la complexité du monde. C'est ce que réussit parfaitement ce très bel ouvrage.

\section{AUTEURS}

\section{CHARLES-FRANÇOIS MATHIS}

Université Bordeaux-Montaigne 